\title{
Simulation, élaboration et caractérisation de cellules photovoltaïques
}

\author{
Céline Ternon $^{\mathrm{a}, \mathrm{b}^{*}}$, Anne Kaminski ${ }^{\mathrm{a}, \mathrm{b}}$, Delphine Constantin ${ }^{\mathrm{b}}$, Lionel Claudon ${ }^{\mathrm{a}, \mathrm{b}}$, Fabien \\ Volpi $^{\mathrm{a}, \mathrm{b}}$, Loïc Vincent ${ }^{\mathrm{b}}$, Quentin Rafhay ${ }^{\mathrm{a}, \mathrm{b}}$, Ahmad Bsiesy ${ }^{\mathrm{b}}$
}

(a) Université Grenoble Alpes, Grenoble INP - Phelma, 3 parvis Louis Néel, BP257, 38016 Grenoble Cedex 1

(b) CIME Nanotech, Pôle CNFM de Grenoble, 3 parvis Louis Néel, 38016 Grenoble Cedex 1

* contact : celine.ternon@grenoble-inp.fr

Avec les besoins croissants en énergie renouvelable, les activités industrielles dans le domaine du photovoltaïque gagnent une place considérable dans le secteur de l'énergie. Afin d'être en adéquation avec ce marché de l'emploi en plein développement, il devient important de former des ingénieurs compétents dans ce domaine.

Un TP a été développé au CIME Nanotech pour simuler, élaborer et caractériser des cellules photovoltaïques de première génération. Le travail de simulation (sur SILVACO ${ }^{\circ}$ ) porte sur les procédés de fabrication de la cellule, ainsi que sur sa réponse I-V sous éclairement. La fabrication (effectuée dans la salle blanche du CIME Nanotech) comprend essentiellement les étapes suivantes: (1) texturation de la surface d'un substrat de silicium (2) réalisation d'une jonction pn, (3) dépôt d'une couche anti-reflet et (4) métallisation. Au-delà de la confrontation aux procédés de fabrication en salle blanche, les étudiants appréhendent l'importance d'étapes technologiques comme, par exemple, la texturation ou la couche anti-reflet ainsi que le rôle de la géométrie, telle que le motif de métallisation. Ces cellules sont ensuite testées électriquement par la mesure des caractéristiques I-V sous obscurité et sous simulateur solaire. Les rendements et autres paramètres caractéristiques (facteur de forme, résistance série...) de chaque cellule sont ensuite calculés et comparés.

Mots-clés: Technologie, salle blanche, cellule photovoltaïque, simulateur solaire, Silvaco®

\section{Introduction}

Le développement de l'énergie photovoltaïque connaît un réel essor depuis une dizaine d'années et de nombreux pôles de recherches y sont maintenant entièrement consacrés. Notamment en Rhône-Alpes où l'INES (Institut National de l'Energie Solaire), localisé à Chambéry, occupe une place de référence dans son domaine à travers l'Europe grâce à des partenaires tels que le CEA ou le CNRS, eux-mêmes à la tête d'un pôle scientifique majeur de rang international à Grenoble. C'est dans l'optique de mieux préparer les élèves-ingénieurs aux enjeux du contexte énergétique actuel que le cas de l'énergie solaire doit être abordé de façon pédagogique, au sein du groupe Grenoble INP.

Le centre de formation CIME Nanotech et l'école d'ingénieur Phelma se donnent pour objectif de proposer des enseignements à l'état de l'art des connaissances scientifiques et des moyens technologiques. Cette pédagogie s'appuie évidemment sur les activités de recherche développées par leurs équipes. Avec cet objectif, il est devenu important de mettre en place des enseignements spécifiques dans le domaine de l'énergie solaire, et plus particulièrement dans celui des cellules solaires photovoltaïques de première génération qui sont les plus répandues de nos jours. Dans cette optique, l'objectif majeur de ce projet réalisé au CIME Nanotech est d'établir une chaîne de procédés technologiques fiable permettant la fabrication de cellules solaires, dans un but pédagogique. 


\section{Objectifs pédagogiques}

Lors de la conception de ce TP, un certain nombre de choix pédagogiques ont été réalisés. En effet, si l'on considère la filière technologique pour les cellules solaires de première génération, il n'y a que peu de points communs avec les filières technologiques de la microélectronique. Or, non seulement le temps en salle blanche pour les étudiants est limité (ce qui contraint la durée des procédés mis en jeu), mais encore le CIME Nanotech dispose essentiellement de moyens dédiés aux micro- et nano-technologies. Il a donc fallu mettre au point un TP permettant d'allier à la fois la découverte des cellules solaires et la découverte des différentes technologies utilisées en salle blanche. Ainsi, des procédés utilisés de façon standard dans l'industrie du photovoltaïque ont été remplacés par des procédés standards de la microélectronique. En particulier, le procédé de sérigraphie couramment utilisé pour la prise de contact électrique disparaît au profit de la photolithographie avec alignement.

En conclusion, les procédés technologiques découverts par les étudiants au cours de ce TP sont les suivants : texturation par bain de $\mathrm{KOH}$, dopage par diffusion, recuit thermique rapide, dépôt par PECVD et pulvérisation cathodique, photolithographie avec alignement, gravure humide, résine à inversion et technique du lift-off. La figure 1 illustre la filière technologique choisie.

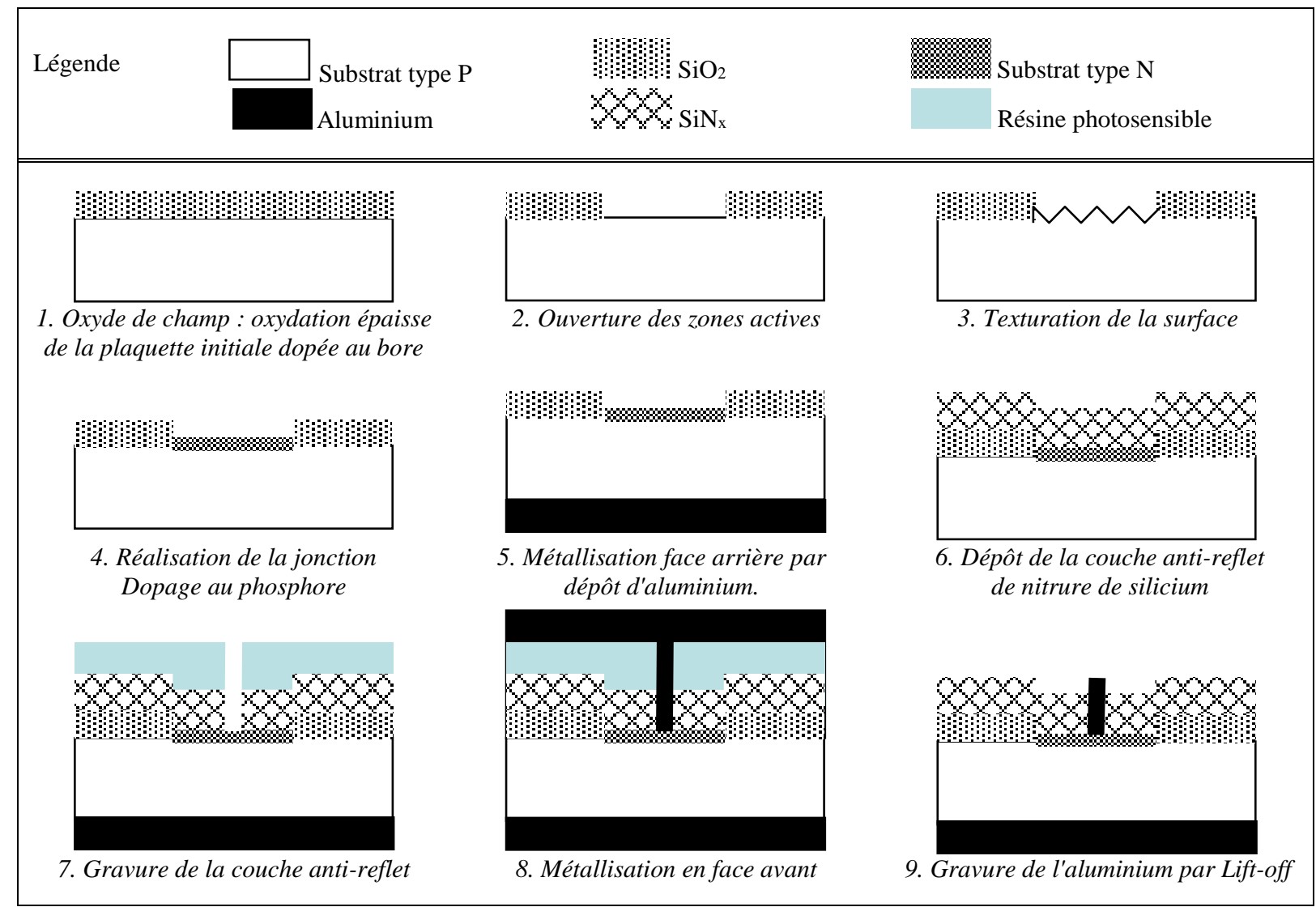

Figure 1: Vue d'ensemble de la filière technologique choisie. Pour plus de clarté, la texturation n'a pas été reportée sur chacun des schémas.

A ces procédés d'élaboration s'ajoute l'initiation aux techniques de caractérisation en ligne telles que l'ellipsométrie, la microscopie électronique à balayage (MEB), la profilométrie et la mesure de résistance carré par la méthode des quatre pointes alignées et la méthode inductive.

En préambule aux séances en salle blanche, une séance d'initiation aux logiciels de simulation ATHENA et ATLAS de la société Silvaco® a été conçue afin de présenter aux 
étudiants les techniques de simulation existantes et de leur permettre de comprendre l'enchaînement des étapes technologiques avant d'y être confronté en salle blanche. Les cellules alors simulées n'intègrent pas l'effet de la couche de texturation.

Enfin, le cycle portant sur les cellules photovoltaïques se termine par une séance de caractérisation. L'objectif de cette séance de caractérisation électrique est d'analyser les performances des cellules photovoltaïques et d'identifier les sources de pertes optiques et électriques. Pour cela, trois types de mesures seront effectuées : caractérisation des cellules solaires sous éclairement, caractérisation des cellules solaires sous obscurité et caractérisation des contacts au moyen de motifs TLM (Transmission Line Model) [1].

\section{Simulation}

Une séance de 4 heures est dédiée à la simulation. Une première étape, réalisée sous ATHENA, permet de simuler la structure de la cellule telle que présentée sur les figures 3(a) et 3(b). Pour parvenir à ce résultat, un certain nombre de paramètres sont fournis aux étudiants, tels que les caractéristiques du substrat initial, les paramètres de diffusion, la longueur d'onde du spectre solaire dont la réflexion doit être minimisée par la couche antireflet... Ainsi, outre la réalisation du programme de simulation, les étudiants doivent également tester les effets de quelques paramètres comme la température de diffusion ou déterminer l'épaisseur de la couche anti-reflet.

Une seconde étape, réalisée sous ATLAS, permet ensuite de modéliser le comportement électrique sous éclairement obtenu avec une telle cellule. La figure 3(c) présente la caractéristique I-V obtenue dans le domaine de fonctionnement actif.

Une fois le programme réalisé, les étudiants sont libres de modifier les différents paramètres, voire éliminer la couche anti-reflet, afin d'en déterminer les conséquences sur les propriétés électriques des cellules.

Enfin, les étudiants n'ayant a priori aucune connaissance sur les cellules solaires, cette séance permet d'introduire les caractéristiques fondamentales telles que le courant de courtcircuit (Icc), la tension en circuit ouvert (Voc), le point de puissance maximum (Pmp), le rendement, le facteur de forme...

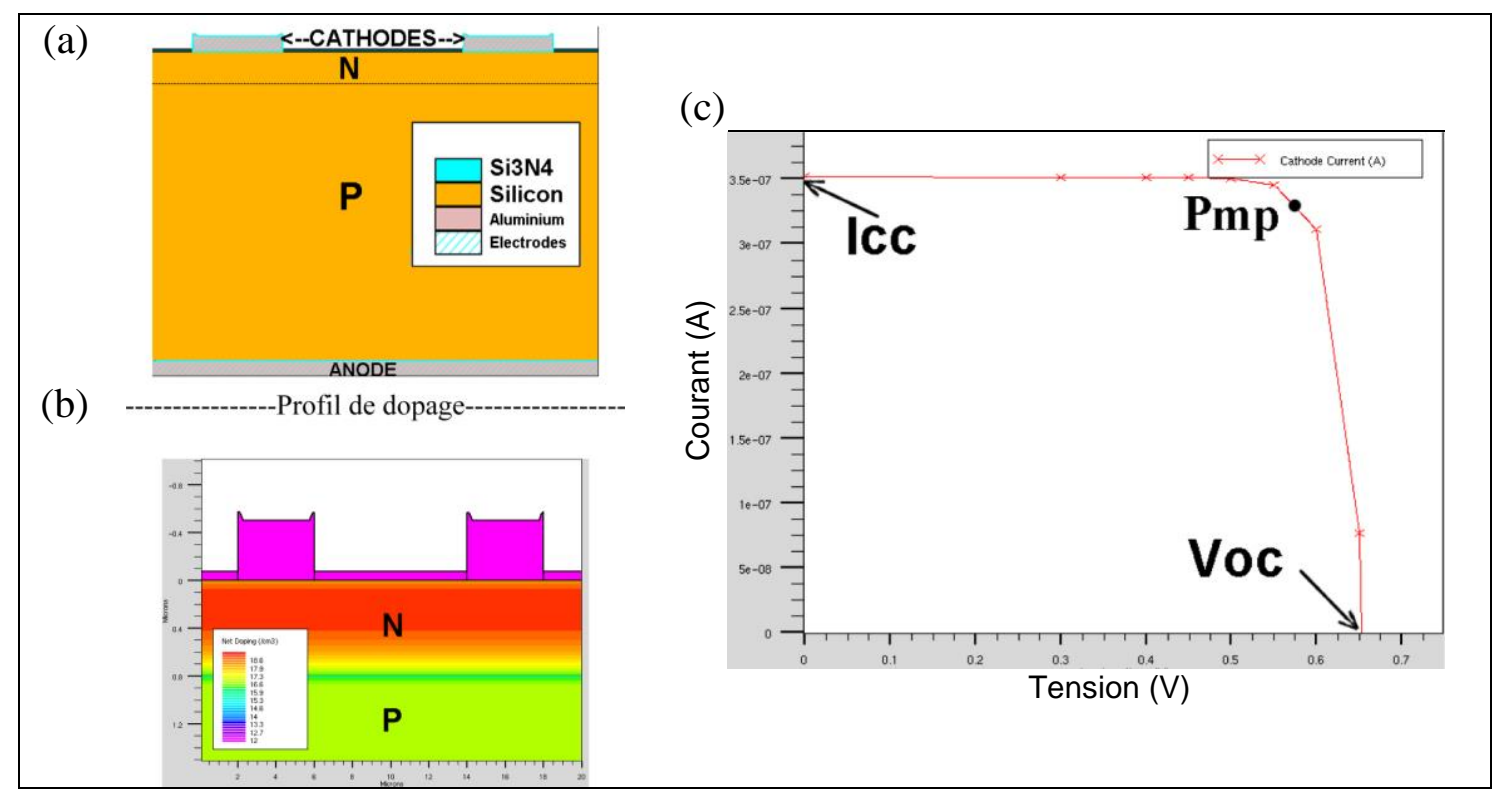

Figure 3 : (a) Structure de la cellule simulée ; (b) Profil de dopage obtenu; (c) Caractéristique I-V obtenue sous éclairement. Icc, courant de court-circuit ; Pmp, point de puissance maximum; Voc, tension en circuit ouvert. 


\section{Réalisation en salle blanche}

Comme évoqué précédemment, cet enseignement «photovoltaïque » développé au CIME Nanotech a pour but de sensibiliser les étudiants aux technologies de la salle blanche en réalisant des cellules photovoltaïques, ce qui implique que certaines étapes technologiques comme la lithographie, peu présentes dans l'industrie photovoltaïque soient néanmoins incluses dans le procédé de fabrication.

A cet effet, un jeu de deux masques de lithographie a été réalisé suivant le schéma présenté sur la figure 4. Le premier niveau de masque, en violet, correspond à l'ouverture des zones actives, le deuxième, en jaune, à la réalisation de la métallisation face avant par lift-off.

Sur une plaque de silicium de deux pouces, les étudiants vont ainsi réaliser trois cellules solaires de $1,5 \mathrm{~cm}$ de côté chacune. La différence entre les trois cellules solaires provient du schéma de métallisation utilisé pour le contact sur la face avant, ce dernier ayant une surface variable d'une cellule à l'autre. Cette différence va se traduire par un taux d'ombrage et une résistance série qui vont varier d'une cellule à l'autre, phénomène que les étudiants pourront analyser au cours de la séance de caractérisation électrique. Les deux motifs restant vont permettre de mesurer la résistivité de contact via des mesures TLM [1].

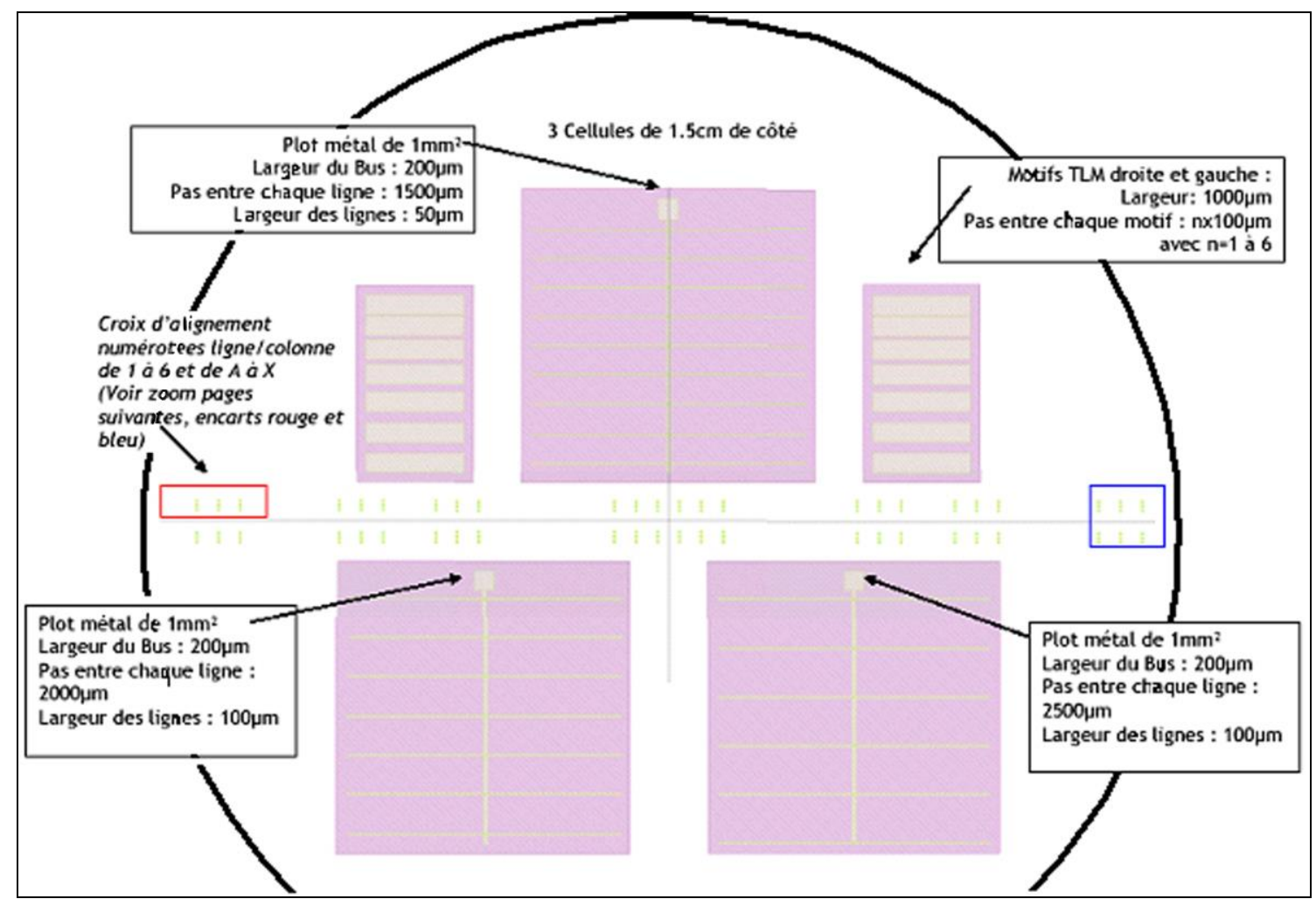

Figure 4 : Masques des cellules solaires réalisées en salle blanche. Le niveau 1, en violet, correspond à l'ouverture des zones actives (rectangles et carrés) dans l'oxyde de champ, le niveau 2, en jaune, à la réalisation des contacts métalliques (peignes et plots de contacts) en aluminium. L'alignement des 2 niveaux est réalisé au moyen des croix d'alignement présentes au milieu du masque. Le cercle noir représente le bord du substrat. 
Le procédé de fabrication des cellules solaires se déroule en 16 heures suivant les étapes suivantes :

- Oxydation thermique humide épaisse sous-traitée par le CIME Nanotech pour réaliser l'oxyde de champ.

- Ouverture des zones actives (masque niveau 1) dans lesquelles seront réalisées les cellules solaires et les motifs de test de résistivité de contact.

- La moitié des plaques est texturée par attaque chimique dans une solution de $\mathrm{KOH}$ diluée. La figure 5 présente une image obtenue par microscopie électronique à balayage (MEB) de la texturation obtenue.

- Nettoyage des plaques ( $\left.\mathrm{HF} ; \mathrm{H}_{2} \mathrm{O}_{2} / \mathrm{H}_{2} \mathrm{SO}_{4} ; \mathrm{HF}\right)$.

- La jonction est ensuite réalisée par diffusion thermique de phosphore dans un four LYDOP.

- Gravure du verre de phosphore et nettoyage des plaques.

- Métallisation face arrière par pulvérisation cathodique d'aluminium.

- Recuit thermique rapide (RTA) du contact pour former un bon contact ohmique en face arrière et un champ arrière passivant par surdopage du silicium à l'aluminium [2].

- Dépôt de la couche antireflet de nitrure de silicium, $\operatorname{SiN}_{\mathrm{x}}$, par PECVD.

- Photolithographie du $\mathrm{SiN}_{\mathrm{x}}$ face avant (masque niveau 2) au moyen d'une résine à inversion pour permettre le lift-off [3, 4].

- Protection de l'aluminium en face arrière par dépôt de résine.

- Gravure du $\mathrm{SiN}_{\mathrm{x}}$ dans une solution $\mathrm{HF} / \mathrm{NH}_{4} \mathrm{~F}$.

- Dépôt d'aluminium face avant par pulvérisation cathodique.

- Gravure du métal en face avant par lift-off.

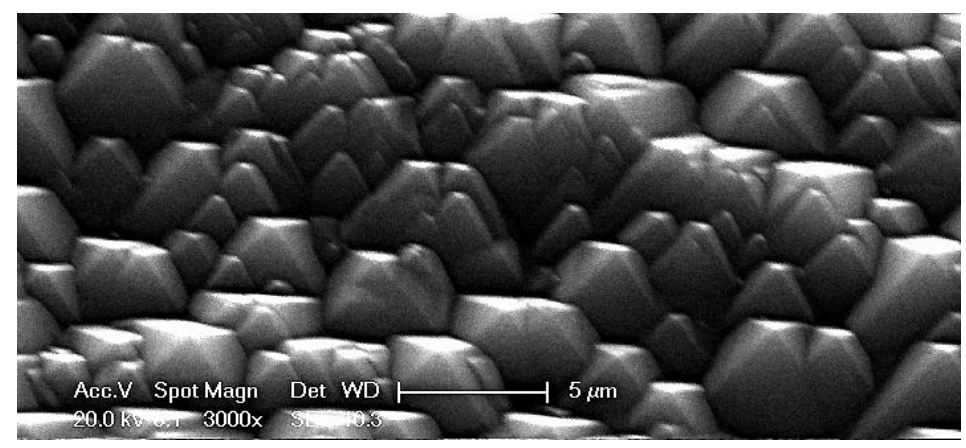

Figure 5 : Texturation de la surface d'un substrat de silicium, observée au MEB Zeiss Ultra+ du CIME Nanotech.

Outre ces étapes technologiques, des étapes de caractérisation en salle blanche sont effectuées : ellipsométrie pour mesurer l'épaisseur et l'indice de l'oxyde de champ et de la couche anti-reflet $\mathrm{SiN}_{\mathrm{x}}$; profilométrie pour mesurer l'épaisseur des différentes couches; mesure par la méthode des quatre pointes et par la méthode inductive de la résistivité du substrat de silicium et de la résistance carré après dopage, mesure par la méthode inductive de la résistance carré de l'aluminium face avant et face arrière, observation au microscope électronique à balayage de la texturation (Fig. 5),... Ces mesures en salle permettent aux étudiants de mieux interpréter les caractérisations finales, effectuées sur cellules 
fonctionnelles, ainsi que de se confronter aux résultats de simulation numérique précédemment obtenus. Ils peuvent ainsi déterminer le dopage du substrat, la résistivité de l'aluminium, comparer les résistances carré visées et obtenues en pratique, ou encore conclure quant à l'efficacité de la couche anti-reflet.

La figure 6 présente le découpage et l'organisation des séances permettant la réalisation des cellules solaires tandis que la structure finale obtenue est présentée sur la figure 7.

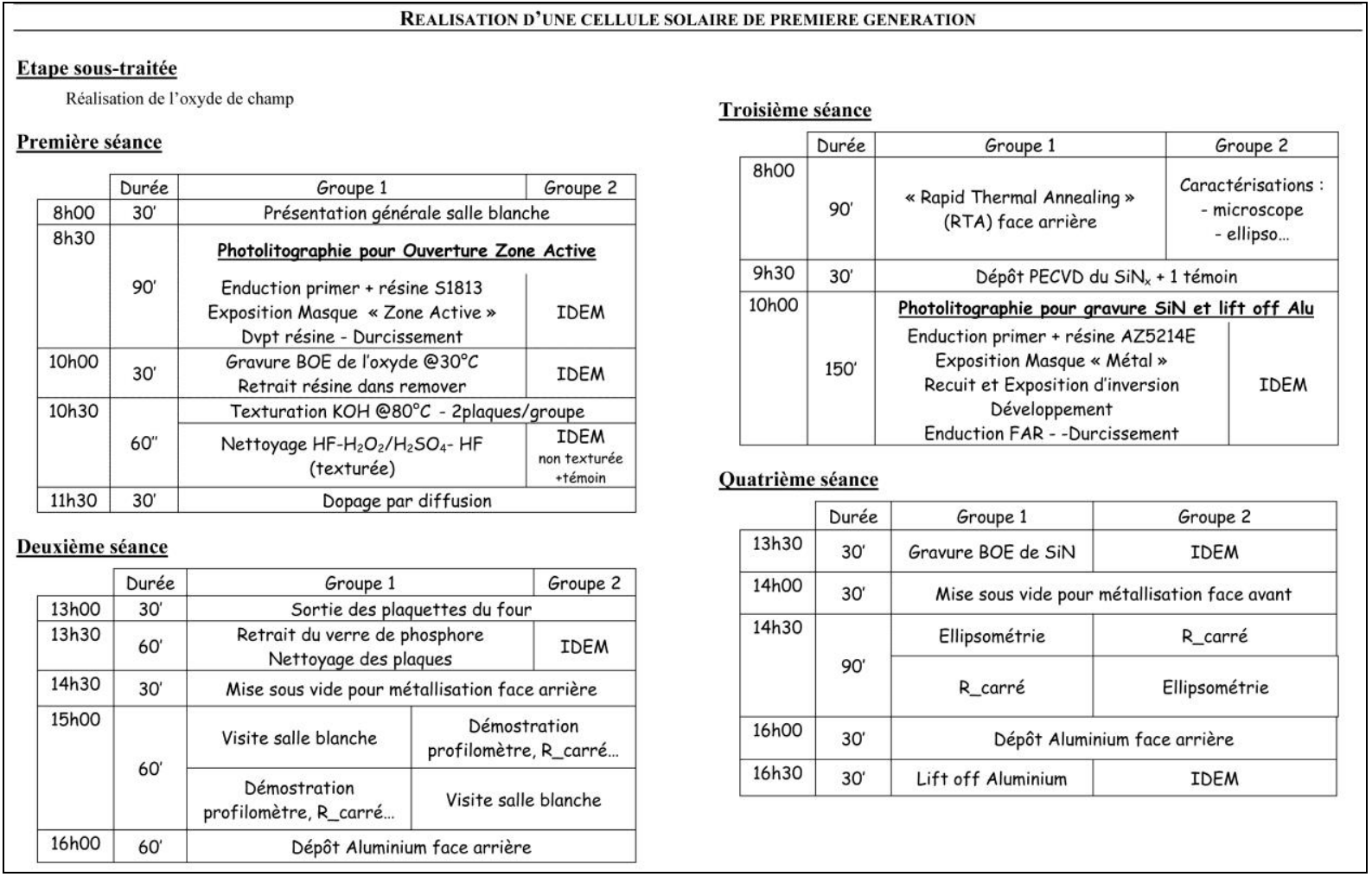

Figure 6 : Découpage des séances indiquant les différentes étapes technologiques ainsi que les caractérisations effectuées en cours de réalisation.

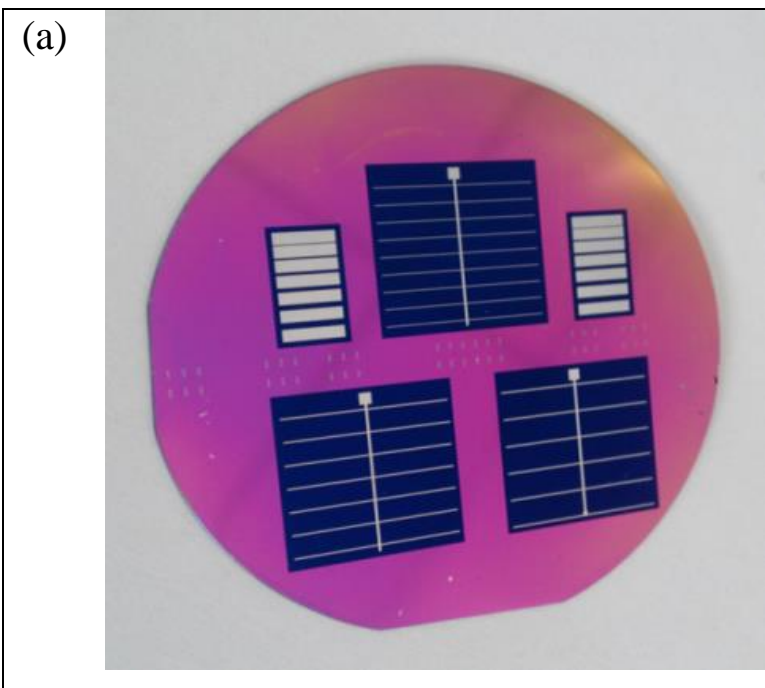

(b)

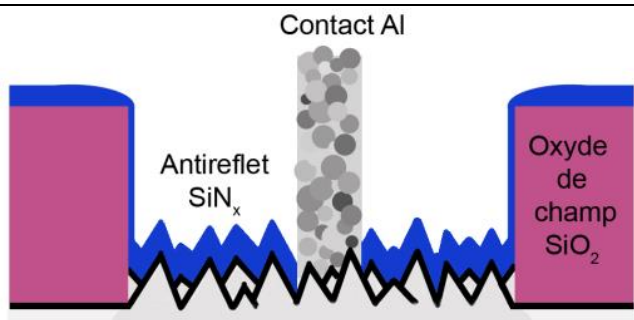

Dopage $\mathrm{N}^{+}$

Substrat P-Si

Dopage $\mathrm{P}++$

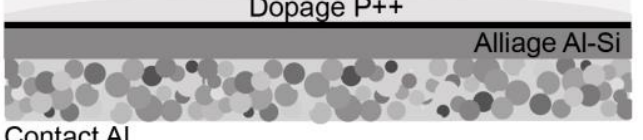

Figure 7 : (a) Photo d'une plaquette à l'issue de toutes les étapes de réalisation. (b) Coupe d'une cellule photovoltaïque au niveau de la ligne de métallisation centrale. 


\section{Caractérisation électrique}

Durant la séance de caractérisation électrique (4 heures), les étudiants vont pouvoir mesurer le rendement des cellules solaires en utilisant le simulateur solaire de classe AAA (fig. 8) acquis par le CIME Nanotech et permettant d'éclairer les cellules solaires avec un rayonnement correspondant à celui du soleil sous AM1.5 (Air Mass 1.5 : rayonnement à la surface de la terre, le soleil faisant un angle d'environ $48^{\circ}$ avec sa position au zénith. Densité de puissance incidente : $1000 \mathrm{~W} / \mathrm{m}^{2}$ ). Ce simulateur solaire utilise un flash lumineux couplé à un système électrique permettant de faire des mesures I-V (en méthode quatre pointes afin de s'affranchir des résistances parasites des pointes et des fils électriques) sous obscurité et sous éclairement.
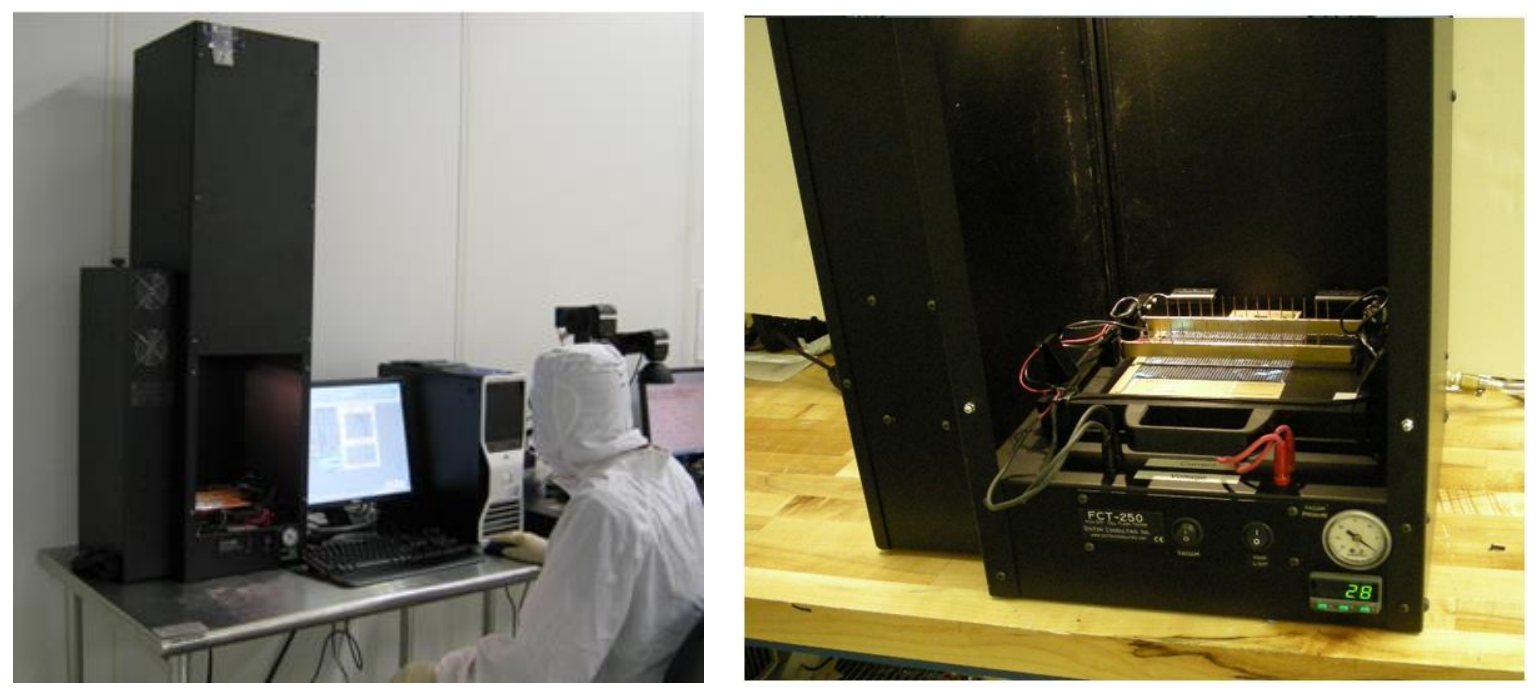

Figure 8: Simulateur solaire et système d'acquisition des courbes I-V

Durant la caractérisation électrique, les étudiants sont sensibilisés à différentes sources de pertes comme les pertes optiques par réflexion (comparaison des performances sur cellules solaires texturées et non texturées en fonction de l'ombrage lié à la métallisation face avant). Les pertes de puissance par effet Joule sont aussi analysées en déduisant la résistance série des mesures I-V pour chaque cellule solaire. Cette mesure est comparée à la résistance série calculée de manière analytique $[1,5,6]$ en tenant compte de la contribution de chaque partie résistive de la cellule solaire à la résistance totale. Pour cela, des mesures complémentaires de résistivité de contact et de résistance carrée de la couche dopée sont réalisées par la méthode TLM [1] (Transmission Line Model) sur un appareil Keithley 4200. Des mesures I-V sont également réalisées sous obscurité afin de calculer les paramètres de la diode (facteur d'idéalité, courant de saturation) ainsi que la résistance parallèle. Le détail des mesures et des calculs demandés est présenté ci-après. 


\section{1- Mesures sur le simulateur solaire :}

1. Mesure de la courbe I-V sous obscurité puis extraction du facteur d'idéalité et du courant de saturation de la diode ainsi que des résistances série, $R_{S}$ et parallèle, $R_{s h}$. Pour cela la formule suivante est utilisée :

$$
\begin{aligned}
& I=I_{s} \exp \left[\underline{q\left(V-R_{s}\right)}\right]+\underline{V-I R_{s}} \quad I_{s}: \text { courant de saturation de la diode } \\
& \mathrm{q} \text { : charge de l'électron } \\
& \mathrm{n} \text { : facteur d'idéalité de la diode } \\
& \mathrm{T} \text { : température } \\
& \mathrm{k} \text { : constante de Boltzman }
\end{aligned}
$$

2. Mesure I-V sous éclairement (fig. 9) des cellules solaires réalisées en salle blanche.

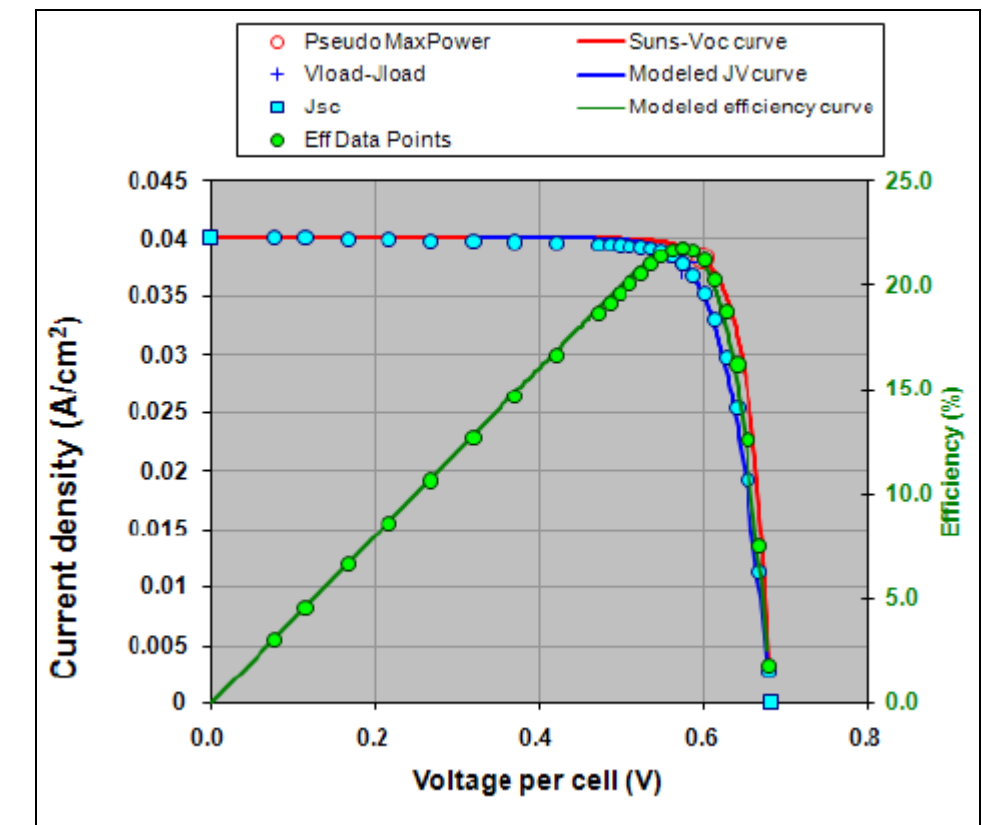

Figure 9 : Courbes I-V expérimentale (cercles bleus), modélisé (courbe bleue), Sun-Voc (courbe rouge) et rendement (courbe verte) [7]

3. A partir de la courbe sous éclairement et de la courbe de puissance (tracée à partir de la courbe I-V mesurée), extraction du courant de court-circuit Isc, de la tension en circuit ouvert Voc, du facteur de forme FF, de la puissance maximale fournie par la cellule solaire Pmax et du rendement de conversion. Le courant sous éclairement s'exprime par la relation :

$$
\mathrm{I}=\mathrm{I}_{\mathrm{L}}-\mathrm{I}_{\mathrm{s}} \exp \left[\frac{\mathrm{q}\left(\mathrm{V}+\mathrm{IR}_{\mathrm{s}}\right)}{\mathrm{nkT}}\right]-\frac{\mathrm{V}+\mathrm{IR}_{\mathrm{s}}}{\mathrm{R}_{\mathrm{sh}}} \quad \mathrm{I}_{\mathrm{L}}: \text { courant photogénéré }
$$

Le rendement de conversion est donné par :

$$
\eta=\frac{P_{\max }}{P_{\text {inc }}}=\frac{\text { FFI }_{\text {sc }} V_{\text {oc }}}{P_{\text {inc }}}
$$

Pinc : puissance incidente

Analyse des résultats en fonction des différents procédés utilisés (avec ou sans texturation...).

4. Pour chaque cellule solaire, calcul de la résistance série en utilisant la courbe « SunVoc » mesurée en même temps que la courbe I-V (fig. 9). Cette courbe correspond à une mesure de la tension en circuit ouvert aux bornes de la cellule à différents éclairements [7]. Les étudiants doivent expliquer la formule utilisée, extraire la résistance série et le rendement sans l'effet de la résistance série.

5. Calcul du taux d'ombre lié aux métallisations pour chaque cellule. 


\section{2-Mesures de résistance de contact (Keithley 4200)}

1. Pour les mesures TLM, les étudiants effectuent les mesures I-V entre chaque plot métallique (fig. 10), extraient la résistance entre deux plots et la tracent en fonction de l'espacement entre les plots (Fig. 10(c)). Ils déduisent de cette courbe la résistance de contact et la résistance carrée de la couche dopée grâce à la relation suivante :

$$
\begin{aligned}
& \mathrm{R}_{\mathrm{t}}(\mathrm{l})=2 \mathrm{R}_{\mathrm{c}}+\frac{\mathrm{R}_{\text {sheet }} 1}{\mathrm{~W}} \quad \begin{array}{l}
\mathrm{R}_{\mathrm{t}} \text { : résistance totale entre deux plots métalliques } \\
1 \text { : espacement entre deux plots métalliques (Fig. 10) } \\
\mathrm{R}_{\text {sheet }} \text { : résistance carré de la couche dopée }
\end{array} \\
& \mathrm{W} \text { : longueur du plot métallique (Fig. 10) }
\end{aligned}
$$

Les étudiants peuvent comparer cette dernière mesure avec celle réalisée en salle blanche par la méthode des quatre pointes.

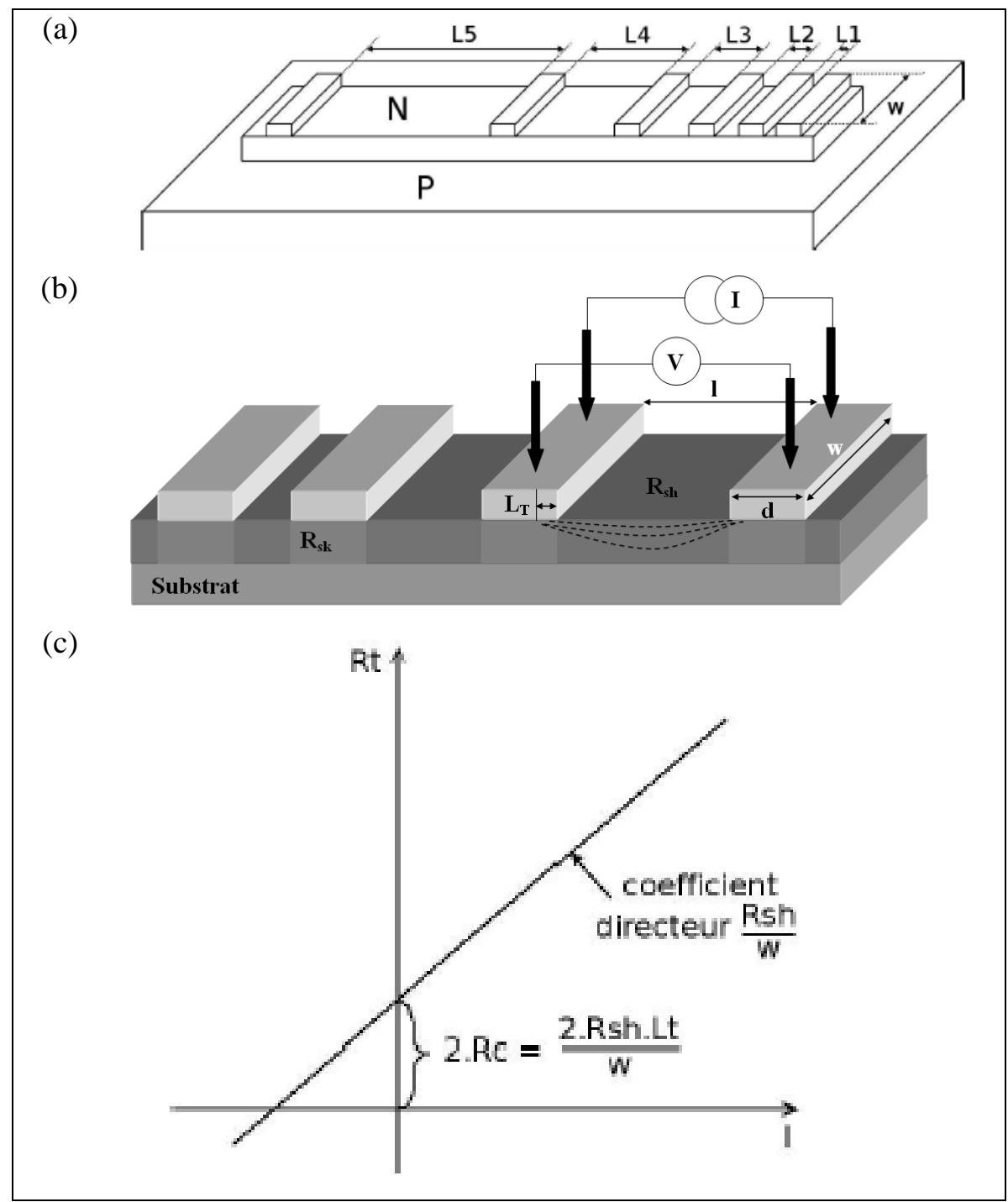

Figure 10: (a) Motif de mesure (plots 1, 2...) avec $L_{5}=5 L_{1}$; (b) Mesure de la résistance entre deux plots; (c) Résistance totale mesurée en fonction de l'espacement entre plots métalliques.

2. A partir des paramètres mesurés, calcul analytique de la résistance liée à la base (substrat de départ), à l'émetteur (dopé $n$ ), à la résistance de la grille face avant, à la résistance de contact des cellules réalisées. Pour cela, l'enseignant guide les étudiants dans leurs calculs. 
La somme de chacune de ces contributions est ensuite comparée à la résistance série extraite de la courbe I-V sous éclairement.

3. Pour finir, les étudiants comparent la contribution des différentes parties de la cellule aux pertes résistives globales et concluent quant aux zones à optimiser tout en tenant compte du taux d'ombre calculé précédemment.

\section{Conclusion et perspectives}

L'enseignement photovoltaïque mis en place au CIME Nanotech, basé sur la simulation, la réalisation et la caractérisation de cellules photovoltaïques de première génération, est actuellement suivi par plusieurs cursus ingénieurs de Phelma. A travers ces travaux pratiques, les étudiants sont sensibilisés à la production d'électricité photovoltaïque mais aussi aux technologies salle blanche et à la caractérisation électrique.

\section{Références}

[1] H.H. Berger, Models for contacts to planar devices, Solid-State Electronics 15, 145-158 (1972).

[2] http://pveducation.org/pvcdrom

[3] Photolithography. Theory and applications, etchants and solvents. Microchemical Editions (2012);

[4] Lithography trouble shooter. Questions and answers around the most common problems in microstructuring. Microchemical Editions (2012).

[5] D. Meier and D. Schröder, Contact resistance : its measurement and relative importance to power loss in a solar cell, IEEE transactions on electron devices 31, 647-653 (1984).

[6] D. Schröder and D. Meier, Solar cells contact resistance : a review, IEEE Transactions on electron devices, 31, 637-647 (1984).

[7] Sinton Instruments : FCT-350 Manual.

Céline Ternon est maître de conférences à Grenoble INP-Phelma depuis 2003 où elle enseigne la physique et la microélectronique. Ses recherches sont centrées autour des nanomatériaux et de leur intégration au sein de dispositifs tels que des détecteurs à ADN ou des cellules photovoltaïques de $3^{\text {ème }}$ génération. C'est cet intérêt pour le photovoltaïque couplé à son implication en salle blanche qui l'a amenée à travailler sur la mise en place d'un TP Photovoltaïque en salle blanche.

Anne Kaminski est professeur à Grenoble INP-Phelma depuis 2010. Au sein de l'école, elle réalise des enseignements concernant le photovoltaïque, la physique mais aussi des travaux pratiques sur les plateformes de simulation, caractérisation et salle blanche du CIME Nanotech. Ses recherches sont réalisées au laboratoire IMEP-LAHC et concernent les cellules photovoltaïques (simulation, élaboration, caractérisation).

Delphine Constantin est ingénieur au CIME Nanotech (Grenoble INP) depuis décembre 2006. Elle est responsable de deux plateformes du service technologique: la salle blanche et la caractérisation électrique. Ses missions s'articulent autour de l'assistance et du soutien à la formation et à la recherche sur ces deux plateformes technologiques et une partie de ses actions concerne la mise au point de nouveaux travaux pratiques (élaboration de la filière technologique et faisabilité) pour la réalisation dispositifs intégrés sur silicium qui font ensuite l'objet de tests électriques et photovoltaïques.

Lionel Claudon a participé à ce projet dans le cadre d'un stage marquant la fin de son Diplôme Universitaire de Technologie en spécialité Mesures Physiques. Ce stage, sous la direction de Céline Ternon, ayant pour objectif la définition d'un protocole de fabrication de 
cellules solaires au sein de la salle blanche du CIME Nanotech à Grenoble. Après avoir intégré l'école Grenoble INP-Ense3, Lionel a obtenu le diplôme d'ingénieur Signal Image Communication Multimédia et travaille maintenant en tant qu'ingénieur logiciel dans l'industrie.

Fabien Volpi est maître de conférences à Grenoble INP-Phelma depuis septembre 2003. Il est co-responsable de la filière Science et Ingénierie des Matériaux, et assure le volet "Formation" du Labex CEMAM (Matériaux Architecturés Multifonctionnels). Il gère aussi l'année 2 du Master International FAME (Matériaux Fonctionnels). Ses enseignements sont axés sur les matériaux fonctionnels (élaboration, intégration, caractérisation), en particulier dans le domaine des microtechnologies. Ses recherches portent sur la caractérisation électrique de matériaux fonctionnels et de structure (diélectriques, métaux, matériaux architecturés,...).

Loïc Vincent est ingénieur à Polytech Grenoble détaché au CIME Nanotech depuis décembre 2007. Il a en charge la responsabilité technique et organisationnelle de la plateforme capteurs et microsystèmes. A ce titre, il a pour activité principale la mise en place et la maintenance de travaux pratiques d'enseignement mais aussi d'apporter un soutien aux travaux de recherche effectués sur la plateforme. De plus, il apporte un soutien technique aux activités de la plateforme caractérisation électrique.

Quentin Rafhay est maître de conférénces à Grenoble INP-Phelma depuis septembre 2010. Il est responsable de la plateforme caractérisation électrique depuis septembre 2010. Dans le cadre de cette activité, il gère la formation des intervenants en caractérisation électrique au CIME, ainsi que les différents investissements relatifs à cette plateforme (renouvellements et mise à jour d'équipements, propositions de nouveaux TP). Ses recherches s'articulent autour de la caractérisation et la modélisation de composants MOSFET.

Ahmad Bsiesy est professeur à l'Université Joseph Fourier-Grenoble I. Il dirige depuis 2008 le CIME Nanotech (Centre Interuniversitaire de Microélectronique et Nanotechnologies), pôle CNFM de Grenoble. Le CIME Nanotech est un centre de ressources et de plateforme technologique qui propose des équipements et des services pour la formation et la recherche en micro- et nanotechnologies. Les travaux de recherche actuels de A. Bsiesy concernent l'élaboration et la caractérisation des structures MIM (Métal-Isolant-Métal) avec oxydes à haute permittivité diélectrique pour des applications en nanoélectronique. 